ARTICLE

\title{
Dynamic transition of current-driven single- skyrmion motion in a room-temperature chiral-lattice magnet
}

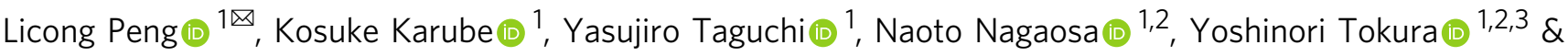
Xiuzhen Yu (D) ${ }^{1 凶}$

Driving and controlling single-skyrmion motion promises skyrmion-based spintronic applications. Recently progress has been made in moving skyrmionic bubbles in thin-film heterostructures and low-temperature chiral skyrmions in the FeGe helimagnet by electric current. Here, we report the motion tracking and control of a single skyrmion at room temperature in the chiral-lattice magnet $\mathrm{Co}_{9} \mathrm{Zn}_{9} \mathrm{Mn}_{2}$ using nanosecond current pulses. We have directly observed that the skyrmion Hall motion reverses its direction upon the reversal of skyrmion topological number using Lorentz transmission electron microscopy. Systematic measurements of the single-skyrmion trace as a function of electric current reveal a dynamic transition from the static pinned state to the linear flow motion via a creep event, in agreement with the theoretical prediction. We have clarified the role of skyrmion pinning and evaluated the intrinsic skyrmion Hall angle and the skyrmion velocity in the course of the dynamic transition. Our results pave a way to skyrmion applications in spintronic devices.

\footnotetext{
${ }^{1}$ RIKEN Center for Emergent Matter Science (CEMS), Wako, Japan. ${ }^{2}$ Department of Applied Physics, University of Tokyo, Bunkyo-ku, Japan. ${ }^{3}$ Tokyo College,

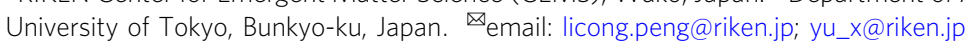


M agnetic skyrmions with vortex-like spin textures can be moved by ultralow current density and hence are promising candidates for information carriers in energyefficient spintronic devices ${ }^{1,2}$. Skyrmions possess topological stability described by the integer character of their topological number $N_{s k}{ }^{3}$

$$
N_{s k}=\frac{1}{4 \pi} \iint \boldsymbol{n} \bullet\left(\frac{\partial \boldsymbol{n}}{\partial x} \times \frac{\partial \boldsymbol{n}}{\partial y}\right) d x d y
$$

where $\boldsymbol{n}=\frac{\boldsymbol{M}}{|\boldsymbol{M}|}$, and $\boldsymbol{M}$ is the magnetization. This topological characteristic imparts skyrmions with particle-like properties, extraordinary metastability 4,5 , and emergent electromagnetic phenomena $^{1-3}$. The flexible shape deformation of skyrmions via the topological protection allows skyrmions to avoid impurities during the current-driven motions, in contrast to domain walls and helices ${ }^{6-8}$. Under electric current excitation, the skyrmion motion is driven by the spin-transfer torque (STT). In a system containing impurities, it can be described by the Thiele equation $6,9,10$

$$
\boldsymbol{G} \times\left(\boldsymbol{v}_{\boldsymbol{s}}-\boldsymbol{v}_{\boldsymbol{d}}\right)+\mathcal{D}\left(\beta \boldsymbol{v}_{\boldsymbol{s}}-\alpha \boldsymbol{v}_{\boldsymbol{d}}\right)+\boldsymbol{F}_{\mathrm{pin}}=0
$$

where $v_{d}=\sqrt{v_{x}^{2}+v_{y}^{2}}$ is the skyrmion drift velocity and $v_{s}$ is the velocity of the conduction electrons. The first term describes the Magnus force represented by the Magnus vector $\boldsymbol{G}=\left(0,0,4 \pi N_{s k}\right)$. The second term corresponds to the dissipative force related to the tensor $\mathcal{D}$, where $\alpha$ is the Gilbert damping factor and $\beta$ is the nonadiabatic coefficient. The third term is the pinning force $\boldsymbol{F}_{\text {pin }}$ arising from impurities. The onset of skyrmion movement is determined by the competition between the underlying pinning sites within the materials and the driving force; that is, skyrmions are pinned by defects and substantially are moved by an external force when it exceeds a certain threshold. Hence the skyrmion motion exhibits a dynamic transition with increasing the driving current, i.e., from the static pinned state to the flow motion by way of a creep motion, as suggested by numerical simulations ${ }^{11,12}$.

The dynamic transition of Néel-type skyrmionic bubbles with micrometer size, which is caused by the spin-orbit torque (SOT), has been demonstrated in thin-film heterostructures with interfacial Dzyaloshinskii-Moriya interaction $(\mathrm{DMI})^{13}$. Driving skyrmionic bubbles requires a high current density of $\sim 10^{10}-10^{12} \mathrm{~A} \mathrm{~m}^{-2}$ to overcome the randomly distributed pinning sites in synthetic multilayered films prepared by magnetron sputtering techniques ${ }^{13-17}$. In contrast, the nanoscale skyrmion dynamics driven by the STT in chiral-lattice magnets is attractive because of the ultralow onset-current density $\left(\sim 10^{6} \mathrm{~A} \mathrm{~m}^{-2}\right)$ to move skyrmions ${ }^{1,2}$. Previous investigations have focused on the nucleation and motion of skyrmion clusters ${ }^{18-20}$ since a single skyrmion is difficult to isolate in the thermodynamic equilibrium phase in chiral-lattice magnets. The first demonstration of a single-skyrmion torque motion has been reported in the FeGe helimagnet at $120 \mathrm{~K}$, well below room temperature $(\mathrm{RT})^{20}$, whereas the control of single-skyrmion motion at RT is valuable for future applications in energy-efficient spintronic devices. However, a direct experimental demonstration of the dynamic transition of the single-skyrmion motion at RT in chiral magnets remains elusive.

Among various chiral-lattice magnets ${ }^{19,21-25}, \mathrm{Co}_{9} \mathrm{Zn}_{9} \mathrm{Mn}_{2}$ that can host RT skyrmions is a good target material for manipulating skyrmions with electric current. $\mathrm{Co}_{9} \mathrm{Zn}_{9} \mathrm{Mn}_{2}$ has a noncentrosymmetric-cubic structure with the space group $P 4_{1} 32$ or $P 4_{3} 32$ (Fig. 1a). The transition temperature $\left(T_{C}\right)$ from the paramagnetic state to the spin-spiral ordered state is $\sim 396 \mathrm{~K}^{24}$, i.e., well above RT, which is essential for avoiding skyrmion annihilation in applications. The equilibrium skyrmion lattice (SkL) occupies a narrow region in the temperature-magnetic field ( $T-B)$ phase diagram, just below $T_{C}{ }^{24}$ Meanwhile, a conical state, which is thermodynamically stable at RT, provides a broad magnetization-polarized background for manipulating isolated skyrmions. Therefore, we choose $\mathrm{Co}_{9} \mathrm{Zn}_{9} \mathrm{Mn}_{2}$ to demonstrate the current-driven single-skyrmion motion at RT.

In this work, we have created a single metastable skyrmion at RT in $\mathrm{Co}_{9} \mathrm{Zn}_{9} \mathrm{Mn}_{2}$. We have directly tracked the single-skyrmion translational and Hall motions induced by nanosecond current pulses. By reversing the pulsed current direction, we could control the trace of the isolated skyrmion. By flipping the magnetic field, thus generating the skyrmion with an opposite sign of a topological number, we demonstrate the opposite Hall motion of the skyrmion transverse to the electric current direction. We also report the current-induced dynamic transition of the singleskyrmion motion from the pinned state to the flow motion via the creep motion, revealing the influence of pinning sites on the skyrmion dynamics.

\section{Results}

Creation of isolated skyrmion in $\mathrm{Co}_{9} \mathrm{Zn}_{9} \mathrm{Mn}_{2}$-based microdevice. We study the single-skyrmion dynamics in a microdevice composed of a (001) $\mathrm{Co}_{9} \mathrm{Zn}_{9} \mathrm{Mn}_{2}$ thin plate (see Fig. 1b and Supplementary Fig. 1). The application of a magnetic field will only generate a conical state, while the $\mathrm{SkL}$ is suppressed at $\mathrm{RT}^{24}$. Therefore we use electric current pulses to create the metastable SkL in the thermodynamically stable conical phase at RT (Supplementary Fig. 2). A single skyrmion is then isolated (Fig. 1c) from the metastable SkL by controlled sweeps of magnetic field (see details in Supplementary Fig. 2). The metastable single skyrmion generated at $-80 \mathrm{mT}$ exhibits a small size of $\sim 100 \mathrm{~nm}$. The negative value of the applied magnetic field means that the field is directed along $-z$ direction, i.e., from the top to the bottom of the sample, which leads to the magnetizations pointing upwards at the skyrmion center and downwards at the periphery (Fig. 1d). Hence, the topological number $N_{s k}$ of the skyrmion is +1 according to its definition in Eq. (1). The magnetic induction field map of the skyrmion (Fig. 1c) indicates a clockwise helicity for the skyrmion, which is encoded by the hue-saturation-lightness color wheel. The surrounding background of the skyrmion displaying dark contrast encodes the polarized magnetization, i.e., the uniform magnetization of a conical state at a small magnetic field of $-80 \mathrm{mT}$. The conical background isolates the skyrmion, which allows avoiding complicated interaction among skyrmions ${ }^{13,26}$. Upon excitation with a pulsed electric current $(j)$ from right to left (as marked by the blue arrow in Fig. 1d), the skyrmion is expected to show a translational motion in the antiparallel direction to the current, and the Hall motion transverse to the current direction (schematically drawn in Fig. 1d).

Current-driven single-skyrmion motions at RT. To study the single-skyrmion dynamics, we have applied nanosecond current pulses one by one to the $\mathrm{Co}_{9} \mathrm{Zn}_{9} \mathrm{Mn}_{2}$-based microdevice and tracked the skyrmion motion at RT. A series of Lorentz transmission electron microscopy (L-TEM) images in Fig. 2 show the single-skyrmion motion driven by sequential 150-ns pulses. The short pulse duration suppresses the skyrmion nucleation/annihilation and the Joule heating effect ${ }^{4,20}$. Upon excitation with an electric current of $j=-6.06 \times 10^{10} \mathrm{~A} \mathrm{~m}^{-2}$ flowing from right to left, the circled single skyrmion moves from the lower-left corner to the upper-right corner of the viewing area (Fig. $2 \mathrm{a}-\mathrm{d}$, see details in Supplementary Movie 1), exhibiting both the translational displacement $(\Delta x)$ and the transverse displacement $(\Delta y)$ of the Hall motion at RT (as indicated in Fig. 2e). Under an electric current of $j=6.32 \times 10^{10} \mathrm{~A} \mathrm{~m}^{-2}$ with an opposite direction, i.e., from left to right, both the translational and transverse displacements of the skyrmion are inverted, as shown in Fig. $2 \mathrm{f}-\mathrm{j}$ (see details in Supplementary Movie 2). The trajectories of the 
a $\quad P 4_{1} 32$

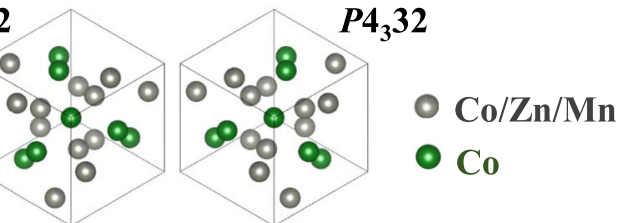

b

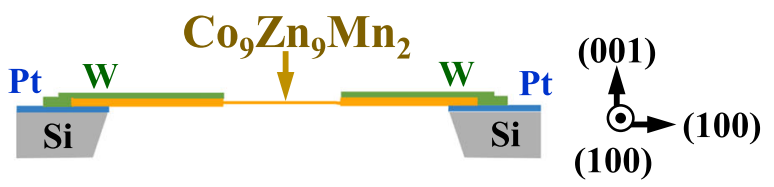

C
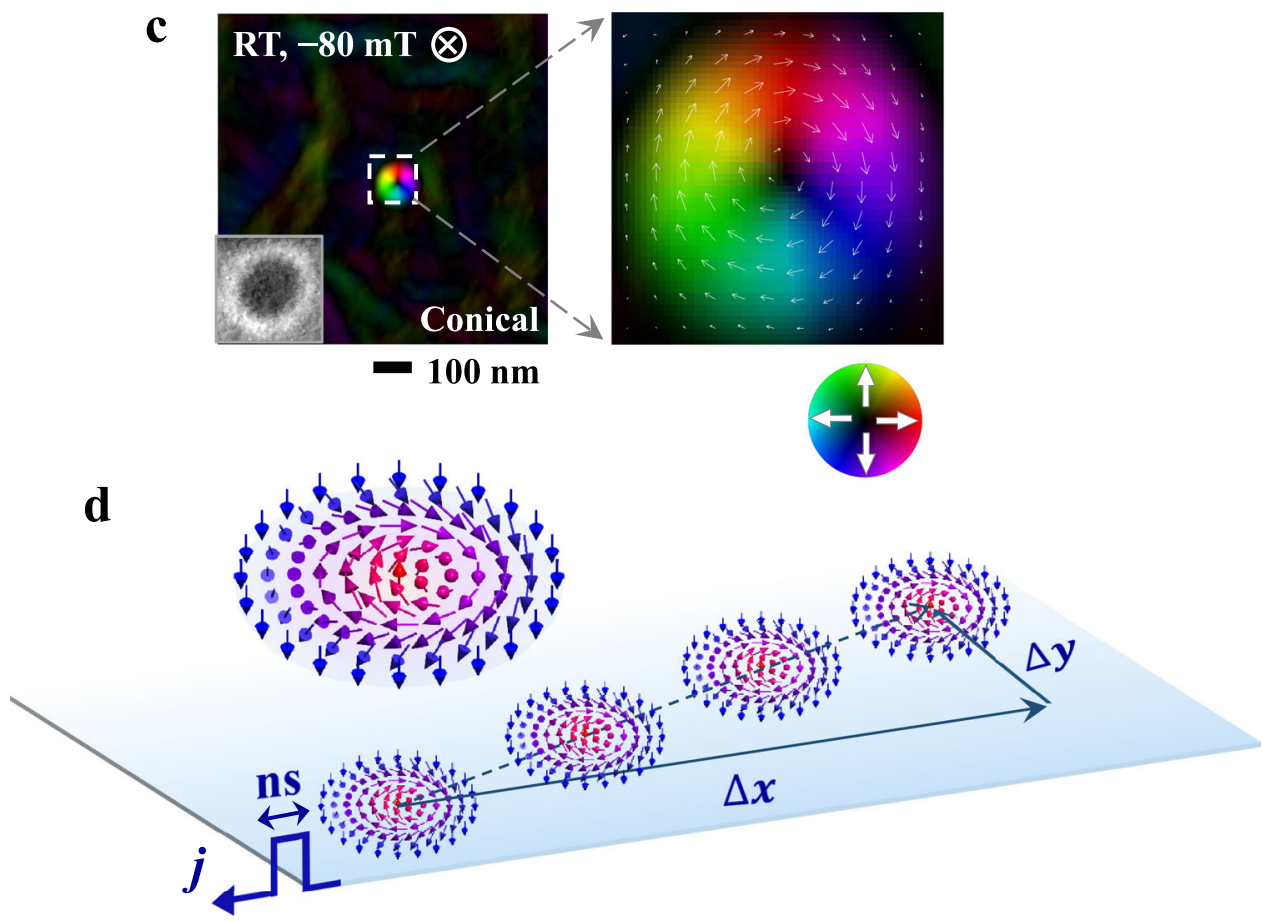

Fig. 1 A single skyrmion in a $\mathbf{C o}_{9} \mathbf{Z} \mathbf{n}_{9} \mathbf{M n}_{\mathbf{2}}$-based microdevice at room temperature (RT). a Schematic of the crystal structure of $\mathrm{Co}_{9} Z n_{9} M_{2}(s p a c e$ group: $P 4_{1} 32$ or $P_{4} 32$ ). $\mathbf{b}$ The cross-section drawing of the microdevice consisting of a (001) $\mathrm{Co}_{9} \mathrm{Zn}_{9} \mathrm{Mn}_{2}$ thin plate (see details in Supplementary Fig. 1). c Magnetic induction maps of a metastable skyrmion generated at RT and a magnetic field of $-80 \mathrm{mT}$ applied along the $-z$-direction. The inset in (c) shows the over-focus L-TEM image of the skyrmion. The color wheel encodes the direction of in-plane magnetic components, and dark contrast encodes the outof-plane components. $\mathbf{d}$ Schematic of skyrmion motion with the translational displacement $(\Delta x)$ and transverse displacement $(\Delta y)$ induced by pulsed electric current $(j)$ flowing from left to right.

single skyrmion, as marked within the L-TEM images of Fig. 2d, i, demonstrate that the straight skyrmion motions is reversed by reversing the current direction (Fig. 2e, j). The abovementioned L-TEM observations indicate an efficient control of singleskyrmion motion by nanosecond current pulses.

Reversal of skyrmion Hall motion. By flipping the direction of the magnetic field applied normally to the thin plate, the topological number is changed from $N_{s k}=+1$ (Fig. 3a) to $N_{s k}=-1$ (Fig. 3f) as defined by Eq. (1). When the electric current with a density $j$ flows along the $x$-axis, the resultant skyrmion velocities $v_{x}$ and $v_{y}$ can be written as ${ }^{6}$,

$$
\begin{gathered}
v_{x}=\frac{\left(4 \pi N_{s k}\right)^{2}+\left(\alpha \mathcal{D}+A / v_{d}\right) \beta \mathcal{D}}{\left(4 \pi N_{s k}\right)^{2}+\left(\alpha \mathcal{D}+A / v_{d}\right)^{2}} \times\left(-\frac{p a^{3}}{2 e M} j\right) \\
v_{y}=\frac{\left(\alpha \mathcal{D}-\beta \mathcal{D}+A / v_{d}\right) 4 \pi N_{s k}}{\left(4 \pi N_{s k}\right)^{2}+\left(\alpha \mathcal{D}+A / v_{d}\right)^{2}} \times\left(-\frac{p a^{3}}{2 e M} j\right)
\end{gathered}
$$

where $p$ is the spin polarization, $a$ is the lattice constant, $e(>0)$ is the elementary charge, and $A$ is the pinning term. Note that Eqs. (3) and (4) are based on the mean-field like treatment of the impurity pinning effect represented by the $A$ term, and hence cannot describe the skyrmion creep motion. The $v_{y}$ is an odd function of $N_{s k}$, and hence the skyrmion Hall motion shows an opposite transverse displacement upon the $N_{s k}$ reversal. On the other hand, since $v_{x}$ is an even function of $N_{s k}$, the translational skyrmion motion does not change its direction with $N_{s k}$. In Fig. $3 \mathrm{~b}-\mathrm{d}$, the over-focus L-TEM images show the $N_{s k}=$ +1 skyrmion with dark contrast at $-80 \mathrm{mT}$ (see details in Supplementary Movie 3). Meanwhile, Fig. 3g-i show the $N_{s k}=-1$ skyrmion with bright contrast in the over-focus LTEM images at $+50 \mathrm{mT}$ with a flipped field direction (see details in Supplementary Movie 4 and Supplementary Fig. 3). The reversed contrast of the skyrmions between Fig. 3b, g indicates the reversed helicity of the skyrmions, in agreement with the expected configurations with fixed DMI and in reversed fields, as shown in Fig. 3a, f. Upon pulsed current stimulation, 



Fig. 2 Motion tracking of the current-driven single-skyrmion at RT. a-d, f-i Over-focus L-TEM images showing the single-skyrmion motion stimulated by a-d negative $\left(j=-6.06 \times 10^{10} \mathrm{~A} \mathrm{~m}^{-2}\right)$ and $\mathbf{f}-\mathbf{i}$ positive $\left(j=6.32 \times 10^{10} \mathrm{~A} \mathrm{~m}^{-2}\right)$ pulsed current with a pulse duration of $150 \mathrm{~ns}$ at $-80 \mathrm{mT}$ and RT. The current flows from right to left for (a-d) and from left to right for (f-i) as marked by the arrows above (a) and (f), respectively. The dashed circles mark the skyrmion locations with the dashed arrows in (d) and (i) showing the directions of skyrmion trajectory. $\mathbf{e}, \mathbf{j}$ Summary of the skyrmion motion along ( $x$-axis) and vertical to ( $y$-axis) the electric current direction: e skyrmion positions at $j=-6.06 \times 10^{10} \mathrm{~A} \mathrm{~m}^{-2}$ obtained from (a-d) and Supplementary Movie 1 , and (j) those at $j=6.32 \times 10^{10} \mathrm{~A} \mathrm{~m}^{-2}$ obtained from (f-i) and Supplementary Movie 2. The straight lines in (e) and (j) are for the Hall angle estimations, i.e., $\frac{\Delta y}{\Delta x}$ for $(\mathbf{e})$ and $\frac{-\Delta y}{-\Delta x}$ for $(\mathbf{j})$.
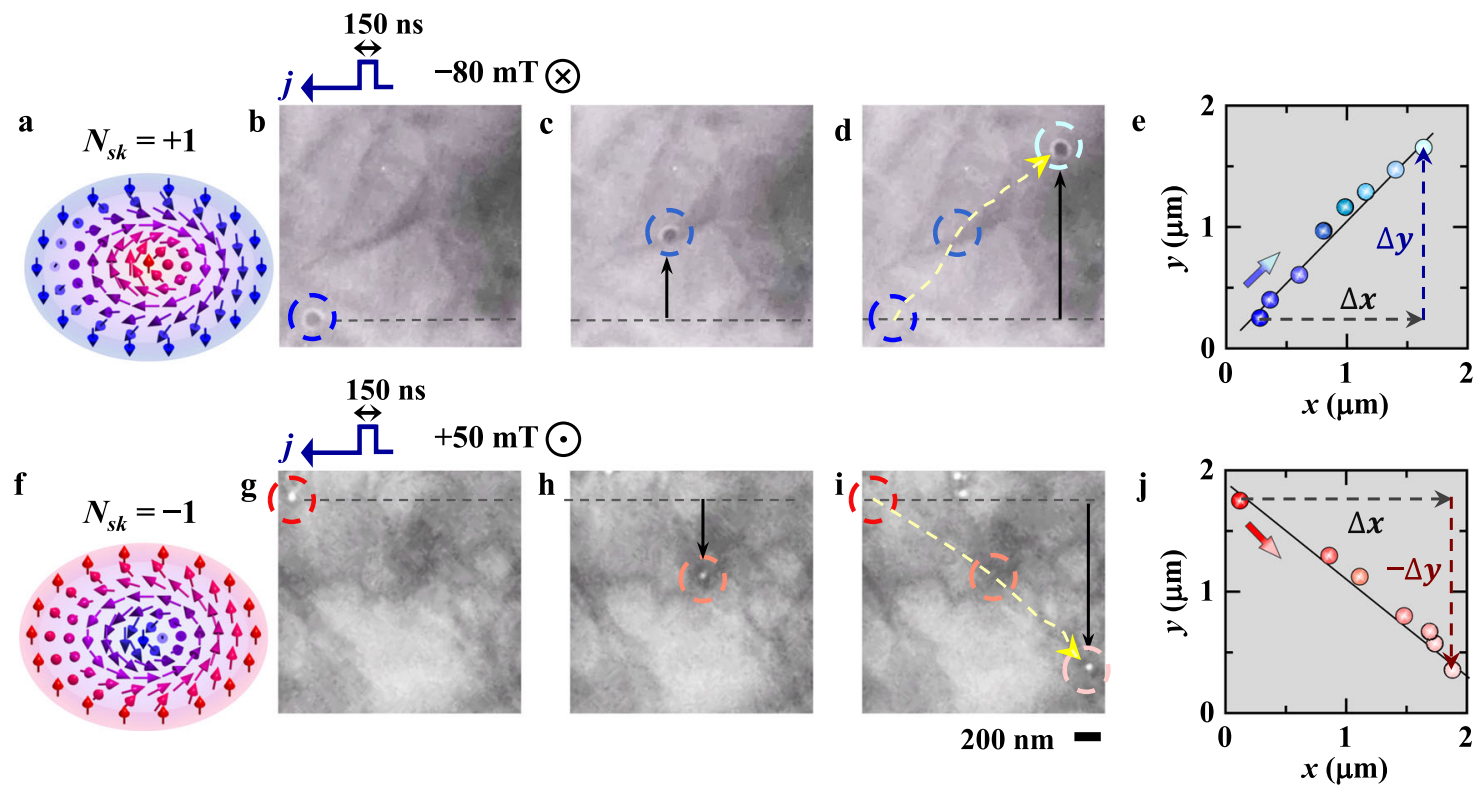

Fig. 3 Reversal of Hall motion for the single skyrmion with an opposite topological number. a, $\mathbf{f}$ Schematics of skyrmions with the topological number $N_{s k}$ of +1 (a) and -1 (f). b-d, $\mathbf{g}$-i Over-focus L-TEM images showing the reversal of Hall-motion direction at RT: $\mathbf{b}-\mathbf{d}$ for the $N_{\text {sk }}=+1$ skyrmion at $-80 \mathrm{mT}$ (as denoted by $\otimes$ ) and at $j=-5.05 \times 10^{10} \mathrm{~A} \mathrm{~m}^{-2}$ and $\mathbf{g}$-i for the $N_{s k}=-1$ skyrmion at $+50 \mathrm{mT}$ (as denoted by $\odot$ ) and at $j=-4.82 \times 10^{10} \mathrm{~A} \mathrm{~m}{ }^{-2}$. The pulsed current flows from right to left as marked by the arrows above (b) and ( $(\mathbf{g})$. The dashed circles mark the skyrmion positions with the arrows in (d) and (i) indicating the direction of the trajectory. $\mathbf{e}, \mathbf{j}$ Summary of the skyrmion traces, with the straight lines showing the translational displacement $(\triangle x)$ and the reversed vertical displacements $(\Delta y$ in $(\mathbf{e})$ and $-\Delta y$ in $(\mathbf{j}))$.

the skyrmion with $N_{s k}=+1$ moves from the lower-left corner to the upper-right corner at $j=-5.05 \times 10^{10} \mathrm{~A} \mathrm{~m}^{-2}$ (Fig. 3b-d), while the skyrmion with $N_{s k}=-1$ moves from the upper-left corner to the lower-right corner at $j=-4.82 \times 10^{10} \mathrm{~A} \mathrm{~m}^{-2}$ (Fig. 3g-i). Figure $3 e, j$ summarize the locations of $N_{s k}=+1$ and -1 skyrmions, respectively, revealing the same direction of their translational motion and the opposite direction of their Hall motion.
We have measured the skyrmion trace slope to quantify the Hall angle $\left(\theta_{s k}\right)$ by linearly fitting the skyrmion locations as follows,

$$
\theta_{s k}=\tan ^{-1} \frac{\Delta y}{\Delta x}
$$

L-TEM observations exemplify the average Hall angle for a pulse duration of $150 \mathrm{~ns}$ and a magnetic field of $-80 \mathrm{mT}$; for example, 

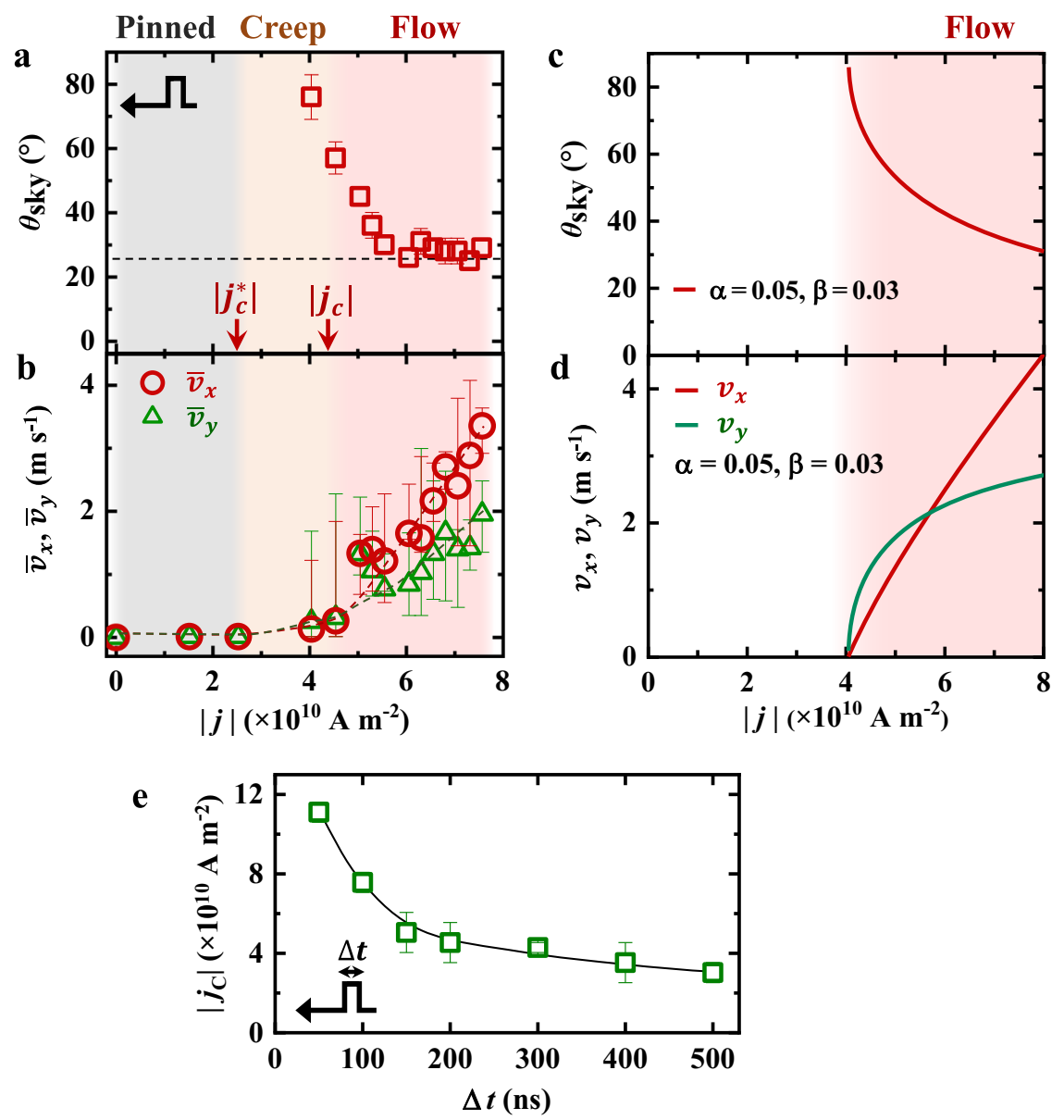

Fig. 4 Dynamic transition of single-skyrmion motion as a function of pulsed electric current. $\mathbf{a}, \mathbf{b}$ Evolution of $\mathbf{a}$ the skyrmion Hall angle $\left(\theta_{s k}\right)$ and $\mathbf{b}$ the average skyrmion velocities $\left(\bar{v}_{x}\right.$ and $\left.\bar{v}_{y}\right)$ with electric current density for $150 \mathrm{~ns}$ pulse duration and $-80 \mathrm{mT}$ magnetic field derived from L-TEM observations. The Hall angle saturates at $\sim 26^{\circ}$ with increasing electric current, as marked by the dashed line in (a). $\bar{v}_{x}$ (red color) and $\bar{v}_{y}($ green color) in $(\mathbf{b})$ are the average transverse and longitudinal velocities of the single skyrmion, respectively. The error bars in (b) show the maximum and minimum velocities for a single current pulse. The gray, orange, and pink regions in $(\mathbf{a}, \mathbf{b})$ correspond to the skyrmion-pinned regime $\left(|j|<\left|j j_{c}^{*}\right| \sim 2.52 \times 10^{10} \mathrm{~A} \mathrm{~m}{ }^{-2}\right)$, the skyrmion creep-motion regime $\left(2.52 \times 10^{10} \mathrm{~A} \mathrm{~m}^{-2}<|j|<4.54 \times 10^{10} \mathrm{~A} \mathrm{~m}^{-2}\right)$, and the skyrmion linear flow-motion regime $\left(|j|>\left|j_{C}\right| \sim 4.54 \times 10^{10} \mathrm{~A} \mathrm{~m}{ }^{-2}\right)$, respectively. c, $\mathbf{d}$ Calculations of $|j|$-dependent (c) Hall angle $\theta_{s k}$ and (d) velocities of $v_{x}$ (red line) and $v_{y}$ (green line) with $\alpha=0.05$ and $\beta=0.03$ for the flow regime of skyrmion motion. e A plot of the critical current density $\left(\left|j_{C}\right|\right)$, driving a single-skyrmion motion into the flow regime, as a function of the pulse duration $\Delta t$.

$\theta_{s k}$ is $\sim 26^{\circ}$ at $j=-6.06 \times 10^{10} \mathrm{~A} \mathrm{~m}^{-2}$ (Fig. 2a-e), while $\theta_{s k}$ is increased to $\sim 45^{\circ}$ with reducing the magnitude of electric current to $j=-5.05 \times 10^{10} \mathrm{~A} \mathrm{~m}^{-2}$ (Fig. 3a-e). L-TEM images of the single-skyrmion motion at various current densities are presented in Supplementary Fig. 4.

Dynamic transition of the single-skyrmion motion. Figure $4 \mathrm{a}, \mathrm{b}$ summarize the $|j|$-dependent skyrmion Hall angle and velocity with the current flowing from left to right. The average values of $\bar{v}_{x}$ and $\bar{v}_{y}$ are estimated from the displacement of the skyrmion after $n$ pulses with the duration of $\Delta t=150 \mathrm{~ns}$ as follows,

$$
\begin{aligned}
& \bar{v}_{x}=\frac{\Delta x}{n \cdot \Delta t} \\
& \bar{v}_{y}=\frac{\Delta y}{n \cdot \Delta t}
\end{aligned}
$$

The error bars in Fig. $4 \mathrm{~b}$ show the maximum and minimum skyrmion velocities for a single-pulse current stimulation. Previous studies ${ }^{11,12,27}$ predict a low depinning force $\left(j_{C}^{*}\right)$ for a creep motion and a relatively high critical electric current $\left(j_{C}\right)$ for a flow motion of skyrmions. Experimentally when we apply a relatively small pulsed current with the magnitude below $2.52 \times 10^{10} \mathrm{~A} \mathrm{~m}^{-2}$, i.e., $\left(|j|<\left|j_{c}^{*}\right|\right.$ ) (as marked in Fig. 4a), the skyrmion does not move, perhaps due to the presence of pinning potential, hence exhibiting a zero velocity (the gray region in Fig. $4 \mathrm{a}, \mathrm{b})$. When slightly increasing the current, i.e., for $\left|j_{c}^{*}\right|$ $\left(2.52 \times 10^{10} \mathrm{~A} \mathrm{~m}^{-2}\right)<|j|<\left|j_{c}\right|\left(4.54 \times 10^{10} \mathrm{~A} \mathrm{~m}^{-2}\right)$, the onset of skyrmion movement is observed: The skyrmion shows a creep motion under sequential current pulses (the orange region in Fig. $4 \mathrm{a}, \mathrm{b}$ ), which leads to a relatively small average velocity below $0.24 \mathrm{~m} \mathrm{~s}^{-1}$. Meanwhile, the $\bar{v}_{y}$ is larger than $\bar{v}_{x}$, i.e., $\frac{\bar{v}_{y}}{\bar{v}_{x}}>1$, hence the calculated Hall angle is rather large at $\sim 76^{\circ}$ for $|j|$ just above $\left|j_{c}^{*}\right|$. When $|j|$ is further increased over $\left|j_{c}\right|\left(4.54 \times 10^{10} \mathrm{~A} \mathrm{~m}^{-2}\right)$, the skyrmion exhibits a linear flow motion (the pink region in Fig. 4a, b), as demonstrated in Figs. 2, 3 and Supplementary Figs. 3, 4, and Supplementary Movies 1-4. With increasing current density, the skyrmion dynamics change from the creep motion to the flow motion, and the Hall angle decreases monotonically and eventually saturates at $\sim 26^{\circ}$ (Fig. 4a). On the other hand, the velocity of the 
single skyrmion increases with the electric current (Fig. 4b). The $\bar{v}_{x}$ increases slightly faster than $\bar{v}_{y}$, leading to $\frac{\bar{v}_{y}}{\bar{v}_{x}}<1$ at $|j|>\left|j_{c}\right|$. The maximum velocities $\bar{v}_{x}$ and $\bar{v}_{y}$ reach $\sim 3.34 \mathrm{~m} \mathrm{~s}^{-1}$ and $\sim 1.95 \mathrm{~m} \mathrm{~s}^{-1}$, respectively, at $|j| \sim 7.57 \times 10^{10} \mathrm{~A} \mathrm{~m}^{-2}$.

Figure $4 \mathrm{c}, \mathrm{d}$ show plots of $|j|$ versus $\theta_{s k}, v_{x}$ and $v_{y}$ using Eqs. (3) and (4) at $\alpha=0.05$ and $\beta=0.03$ (see details in Methods). The fitting curves represent the skyrmion dynamics in a flow regime. As $|j|$ increases, $v_{x}$ (red line in Fig. $4 \mathrm{~d}$ ) and $v_{y}$ (green line in Fig. 4d) increase monotonically, and the $\theta_{s k}$ (Fig. 4c) decreases and tends to approach a saturated value. As $|j|$ decreases, the skyrmion velocity decreases, and the calculated $\theta_{s k}$ shows a rapid increase due to the impurity pinning effect, as the skyrmion is deflected by the Magnus force when the skyrmion approaches defects such as a grain boundary ${ }^{9,11}$. The calculated profiles of $|j|-\theta_{s k},|j|-v_{x}$, and $|j|-v_{y}$ (Fig. 4c, d) coincide with our present in situ L-TEM observations for single-skyrmion dynamics in the flow-motion regime.

Let us move onto the pulse-duration dependence of the skyrmion flow motion (Fig. 4e). At a large $\Delta t$ of $500 \mathrm{~ns}$, an electric current density above $\left|j_{c}\right|$ of $3.03 \times 10^{10} \mathrm{~A} \mathrm{~m}^{-2}$ can induce the skyrmion flow motion; this density is two orders of magnitude smaller than that needed for driving ferromagnetic domain walls ${ }^{28}$. As $\Delta t$ is reduced to $50 \mathrm{~ns}$, the $\left|j_{c}\right|$ is increased up to $1.1 \times 10^{11} \mathrm{~A} \mathrm{~m}^{-2}$. A large $\Delta t$ above $\sim 1 \mathrm{~ms}$, as previously discussed for the FeGe helimagnet ${ }^{20}$, will result in a significant Joule heating effect. The short pulse duration of the nanosecond used in this work reduces the Joule heating and hence promotes the STT effect for the current-driven skyrmion dynamics.

\section{Discussion}

Our systematic measurements demonstrate the single-skyrmion motion as a function of electric current at RT in the chiral-lattice magnet $\mathrm{Co}_{9} \mathrm{Zn}_{9} \mathrm{Mn}_{2}$. Under the pulsed current stimulation, the skyrmion exhibits a trapping-limited motion: it moves during the short current pulse and then is trapped by nearby pinning sites after the stimulus, where the skyrmion can deform, as exemplified by Fig. 2c, d and Supplementary Fig. 4m. The L-TEM captures the two static skyrmion states before/after the current pulse, while the intermediate state during the pulse could not be detected with our experimental setup, suggesting that the skyrmion velocity may be larger than measured values. The measured $\bar{v}_{x}$ reaches $\sim 3.34 \mathrm{~m} \mathrm{~s}^{-1}$ at $|j|=7.57 \times 10^{10} \mathrm{~A} \mathrm{~m}^{-2}$ (Fig. $4 \mathrm{~b}$ ), nearly consistent with our calculations (Fig. 4d). The Hall angle is rather large in the creepmotion regime since the skyrmion is easily trapped by pinning sites at a small $|j|$ just above $\left|j_{C}^{*}\right|$. In contrast, at $|j|>\left|j_{C}\right|$, the skyrmion motion depends less on the pinning sites, demonstrating a saturated Hall angle of $\sim 26^{\circ}$ in $\mathrm{Co}_{9} \mathrm{Zn}_{9} \mathrm{Mn}_{2}$, which is determined by material parameters ${ }^{11,13,20}$. The plot of $|j|-\theta_{s k}$ (Fig. 4a) observed experimentally fits well with our calculations (Fig. 4c) for the STTinduced Bloch-type skyrmion motion in chiral-lattice magnets; in fact, the skyrmion dynamics observed here are in contrast to the SOT-induced Néel-type skyrmion motion in heterostructured thin films, where the $\theta_{s k}$ is close to zero for a small $j$ and exhibits a monotonic increase with increasing the driving force ${ }^{13}$. The mechanism of the spin-polarized electric current acting on skyrmions, i.e., the STT in chiral-lattice magnets or the SOT in heterostructured thin films, may determine the skyrmion dynamic behavior in the system with impurities.

In summary, we have experimentally revealed the transition of skyrmion dynamics from the pinned state to the flow motion via a creep motion as a function of electric current in $\mathrm{Co}_{9} \mathrm{Zn}_{9} \mathrm{Mn}_{2}$, and directly demonstrated the reversal of skyrmion Hall motion with an opposite topological number. Our achievements provide evidence of STT-driven skyrmion dynamics upon nanosecond-pulse current excitation in chiral-lattice magnets, deepen the understanding of the pinning effect on skyrmion dynamics, and will promote the studies of dynamical motions of various topological spin textures.

\section{Methods}

Microdevice preparation. Single crystals of $\mathrm{Co}_{9} \mathrm{Zn}_{9} \mathrm{Mn}_{2}$ were grown by the Bridgman method and examined by X-ray diffraction. The microdevice was prepared using a focused ion beam (FIB) system equipped with a gallium ion gun (NB-5000, Hitachi, Japan). It consisted of a (001) $\mathrm{Co}_{9} \mathrm{Zn}_{9} \mathrm{Mn}_{2}$ thin plate whose left and right edges were connected to two Pt leads using tungsten (W) layers. The top and bottom edges were additionally padded by amorphous carbon (see Supplementary Fig. 1). The orientation of the $\mathrm{Co}_{9} \mathrm{Zn}_{9} \mathrm{Mn}_{2}$ thin plate was checked via a selected area diffraction pattern.

L-TEM observations. Real-space observations of skyrmions were performed using JEM-2100F and JEM-2800F microscopes (JEOL, Japan) at an acceleration voltage of $200 \mathrm{kV}$. A magnetic field was applied normally to the thin plate, and its magnitude was controlled by changing the objective-lens current of the TEM. The L-TEM images under the magnetic field applied along the $+z$-direction were recorded with the JEM-2800F microscope, and the field strength was measured by a Hall probe. The L-TEM images under the magnetic field applied along the $-z$ direction were recorded in the JEM-2100F microscope. The value of nanosecond pulsed current was calculated by dividing the pulsed voltage by sample resistance and sample cross-section area. The pulsed voltage was applied by an arbitrary function generator (AFG 31252, Tektronix, U.S.). Quantitative in-plane induction field maps were obtained by analyzing the under- and over-focus L-TEM images using the software package QPt (HREM Co., Japan) based on the transport-ofintensity equation (TIE) ${ }^{29}$.

Calculations. The calculations of the skyrmion Hall angle and velocity (Fig. 4c, d) are based on the Thiele Eq. (2) in the presence of impurities as given in ref. ${ }^{6}$. The drift velocity of a skyrmion $v_{d}$ is given by

$$
v_{d}=\frac{\sqrt{(\mathcal{D} \alpha A)^{2}+\left(\left(4 \pi N_{s k}\right)^{2}+(\mathcal{D} \alpha)^{2}\right)\left(\left(\beta^{2} \mathcal{D}^{2}+\left(4 \pi N_{s k}\right)^{2}\right) v_{s}{ }^{2}-A^{2}\right)}}{\left(4 \pi N_{s k}\right)^{2}+(\mathcal{D} \alpha)^{2}}
$$

where $v_{s}=-\frac{p a^{3}}{2 e m} j$ is the velocity of the conduction electrons. The parameters are given as follows ${ }^{6,30}$ : dissipative force $\mathcal{D}=5.577 \pi$, damping factor $\alpha=0.05$, nonadiabatic coefficient $\beta=0.03$, pinning term $A \equiv 4 \pi v_{\text {pin }}$ with the pinning velocity $v_{\text {pin }}=3.2$, lattice constant $a=6.32 \mathrm{~A}$, local magnetic moment $M=1$, and spin polarization $p=0.1$ which was estimated from the ratio of magnetizations in the skyrmion phase to the saturated magnetization at $2 \mathrm{~K}^{24,30}$.

\section{Data availability}

The data that support the findings of this study are available from the corresponding author upon reasonable request.

Received: 24 July 2021; Accepted: 3 November 2021; Published online: 24 November 2021

\section{References}

1. Jonietz, F. et al. Spin transfer torques in $\mathrm{MnSi}$ an ultralow current density. Science 330, 1648-1651 (2010).

2. Neubauer, A. et al. Topological Hall effect in the A phase of MnSi. Phys. Rev. Lett. 102, 186602 (2009).

3. Nagaosa, N. \& Tokura, Y. Topological properties and dynamics of magnetic skyrmions. Nat. Nanotechnol. 8, 899-911 (2013).

4. Oike, H. et al. Interplay between topological and thermodynamic stability in a metastable magnetic skyrmion lattice. Nat. Phys. 12, 62-66 (2016)

5. Karube, K. et al. Robust metastable skyrmions and their triangular-square lattice structural transition in a high-temperature chiral magnet. Nat. Mater. 15, 1237-1242 (2016).

6. Iwasaki, J., Mochizuki, M. \& Nagaosa, N. Universal current-velocity relation of skyrmion motion in chiral magnets. Nat. Commun. 4, 1463 (2013).

7. Koshibae, W. \& Nagaosa, N. Theory of current-driven skyrmions in disordered magnets. Sci. Rep. 8, 6328 (2018).

8. Iwasaki, J., Mochizuki, M. \& Nagaosa, N. Current-induced skyrmion dynamics in constricted geometries. Nat. Nanotechnol. 8, 742-747 (2013).

9. Iwasaki, J., Koshibae, W. \& Nagaosa, N. Colossal spin transfer torque effect on skyrmion along the edge. Nano Lett. 14, 4432-4437 (2014).

10. Masell, J. \& Everschor-Sitte, K. Current-induced dynamics of chiral magnetic structures: creation, motion, and applications. https://doi.org/10.1007/978-3030-62844-4_7 (2020). 
11. Reichhardt, C., Reichhardt, C. J. O. \& Milosevic, M. V. Statics and dynamics of skyrmions interacting with pinning: a review. Preprint at arXiv:2102.10464v1 (2021).

12. Reichhardt, C. \& Reichhardt, C. J. O. Thermal creep and the skyrmion Hall angle in driven skyrmion crystals. J. Phys. Condens. Matter 31, 07LT01 (2019).

13. Jiang, W. J. et al. Direct observation of the skyrmion Hall effect. Nat. Phys. 13, 162-169 (2017).

14. Woo, S. et al. Observation of room-temperature magnetic skyrmions and their current-driven dynamics in ultrathin metallic ferromagnets. Nat. Mater. 15, 501-506 (2016).

15. Jiang, W. J. et al. Blowing magnetic skyrmion bubbles. Science 349, 283-286 (2015).

16. Woo, S. et al. Spin-orbit torque-driven skyrmion dynamics revealed by timeresolved X-ray microscopy. Nat. Commun. 8, 15573 (2017).

17. Fert, A., Reyren, N. \& Cros, V. Magnetic skyrmions: advances in physics and potential applications. Nat. Rev. Mater. 2, 17031 (2017).

18. Yu, X. Z. et al. Current-induced nucleation and annihilation of magnetic skyrmions at room temperature in a chiral magnet. Adv. Mater. 29, 1606178 (2017).

19. Yu, X. Z. et al. Skyrmion flow near room temperature in an ultralow current density. Nat. Commun. 3, 988 (2012).

20. $\mathrm{Yu}, \mathrm{X}$. Z. et al. Motion tracking of 80 -nm-size skyrmions upon directional current injections. Sci. Adv. 6, eaaz9744 (2020)

21. Mühlbauer, S. et al. Skyrmion lattice in a chiral magnet. Science 323, 915-919 (2009).

22. Seki, S., Yu, X. Z., Ishiwata, S. \& Tokura, Y. Observation of skyrmions in a multiferroic material. Science 336, 198-201 (2012).

23. Tokunaga, Y. et al. A new class of chiral materials hosting magnetic skyrmions beyond room temperature. Nat. Commun. 6, 7638 (2015).

24. Karube, K. et al. Metastable skyrmion lattices governed by magnetic disorder and anisotropy in $\beta$-Mn-type chiral magnets. Phys. Rev. B 102, 064408 (2020).

25. Peng, L. C. et al. Relaxation dynamics of zero-field skyrmions over a wide temperature range. Nano Lett. 18, 7777-7783 (2018).

26. Lin, S. Z., Reichhardt, C., Batista, C. D. \& Saxena, A. Particle model for skyrmions in metallic chiral magnets: dynamics, pinning, and creep. Phys. Rev. B 87, 214419 (2013).

27. Luo, Y. et al. Skyrmion lattice creep at ultra-low current densities. Commun. Mater. 1, 83 (2020).

28. Parkin, S. S. P., Hayashi, M. \& Thomas, L. Magnetic domain-wall racetrack memory. Science 320, 190-195 (2008).

29. Ishizuka, K. \& Allman, B. Phase measurement of atomic resolution image using transport of intensity equation. J. Electron Microsc. 54, 191-197 (2005).

30. Preißinger, M. et al. Vital role of magnetocrystalline anisotropy in cubic chiral skyrmion hosts. npj Quan. Mater. 6, 65 (2021).

\section{Acknowledgements}

We thank Konstantin V. Iakoubovskii, Wataru Koshibae, Jan Masell, Fumitaka Kagawa, Fehmi S. Yasin, and Mari Ishida for enlightening discussions. We thank Tomoka Kikitsu and Daisuke Hashizume (Materials Characterization Support Team in the RIKEN Center for Emergent Matter Science) and RIKEN CEMS Emergent Matter Science Research Support Team for technical supports with TEM (JEM-2100F) and FIB (NB-5000, Hitachi), respectively. This work was partly supported by Grants-In-Aid for Scientific Research (A) (Grant No.19H00660) from JSPS, and Japan Science and Technology Agency CREST (Grant No. JPMJCR1874 and No. JPMJCR20T1) from JST.

\section{Author contributions}

L.P., Y.Taguchi, Y.Tokura, and X.Y. jointly conceived the project. L.P. designed and fabricated the microdevice, performed the L-TEM observations, and analyzed the experimental results with X.Y. L.P. performed the calculations with N.N. K.K. synthesized the $\mathrm{Co}_{9} \mathrm{Zn}_{9} \mathrm{Mn}_{2}$ crystal with Y.Taguchi. L.P. wrote the manuscript with inputs and comments from all authors.

\section{Competing interests}

The authors declare no competing interests.

\section{Additional information}

Supplementary information The online version contains supplementary material available at https://doi.org/10.1038/s41467-021-27073-2.

Correspondence and requests for materials should be addressed to Licong Peng or Xiuzhen Yu.

Peer review information Nature Communications thanks the anonymous reviewer(s) for their contribution to the peer review of this work. Peer reviewer reports are available.

Reprints and permission information is available at http://www.nature.com/reprints

Publisher's note Springer Nature remains neutral with regard to jurisdictional claims in published maps and institutional affiliations.

(c) (i) Open Access This article is licensed under a Creative Commons BY Attribution 4.0 International License, which permits use, sharing, adaptation, distribution and reproduction in any medium or format, as long as you give appropriate credit to the original author(s) and the source, provide a link to the Creative Commons license, and indicate if changes were made. The images or other third party material in this article are included in the article's Creative Commons license, unless indicated otherwise in a credit line to the material. If material is not included in the article's Creative Commons license and your intended use is not permitted by statutory regulation or exceeds the permitted use, you will need to obtain permission directly from the copyright holder. To view a copy of this license, visit http://creativecommons.org/ licenses/by/4.0/.

(c) The Author(s) 2021 